\title{
Parámetros cinéticos de espermatozoides en semen fresco y crioconservado de alpaca
}

(Vicugna pacos L.)

\section{Kinetic parameters of sperm in fresh and cryopreserved semen of alpaca (Vicugna pacos L.)}

\author{
Apaza-Callisaya Blanca Nieves $^{1 *}$, Loza-Murguia Manuel Gregorio ${ }^{1,2} \odot$, Quispe-Paxipati Cesar Humberto ${ }^{1}$,
} Machicado-Gómez Richard Max ${ }^{1}$, Achu-Nina Cristóbal ${ }^{1}$

\begin{tabular}{l}
\hline Datos del Artículo \\
\hline${ }^{1}$ Universidad Católica Boliviana San Pablo- \\
UCBSP. Unidad Académica Campesina \\
Tiahuanacu UAC-T. Ingeniería Zootécnica. \\
Km 74 . Carretera Internacional La Paz- \\
Desaguadero. \\
Tel +591-2-2895100. \\
La Paz, Estado Plurinacional de Bolivia. \\
2 Departamento de Enseñanza e Investiga \\
ción en Bioquímica \& Microbiología- \\
DEI\&BM. Unidad Académica Campesina \\
Tiahuanacu-UAC-T. \\
Direción de contacto: \\
Universidad Católica Boliviana San Pablo- \\
UCBS. Unidad Académica Campesina \\
Tiahuanacu UAC-T. Ingeniería Zootécnica. \\
Km 74. Carretera Internacional La Paz- \\
Desaguadero. \\
La Paz, Estado Plurinacional de Bolivia. \\
Tel: +591-75826254. \\
Blanca Nieves Apaza-Callisaya \\
E-mail address: \\
brisaclara@ @otmail.com \\
\hline Palabras clave: \\
Semen, \\
Semen, \\
ASAC, \\
evaluación, \\
crioconservación, \\
alpaca, \\
parámetros cinéticos. \\
\hline
\end{tabular}

J. Selva Andina Anim. Sci. 2020; 7(1):17-29.

\section{Historial del artículo.}

Recibido mayo, 2018

Devuelto octubre 2018

Aceptado diciembre, 2019

Disponible en línea, abril, 2020

\section{Editado por:}

Selva Andina

Research Society
(C) 2020. Journal of the Selva Andina Animal Science. Bolivia. Todos los derechos reservados.

La crioconservación de los epz de alpaca es importante para la mejora genética. El presente trabajo tuvo como propósito evaluar los parámetros cinéticos de spz en semen fresco y crioconservado en dilutores, mediante el equipo ASAC. Se utilizaron 3 alpacas machos, que fueron entrenados durante un mes en la colección de semen con la vagina artificial. El esperma se congeló empleando dos diluyentes (AndroMed comercial) y (AndroMed comercial, ácido cítrico, gen-

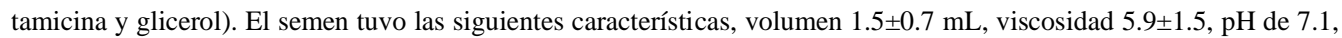
con tendencia a alcalinidad y el color que predominó fue el blanco translucido. Los promedios de parámetros cinéticos en semen fresco fueron VCL $(25.8 \pm 4.2 \mu \mathrm{m} / \mathrm{s})$, VSL $(10.3 \pm 2.5 \mu \mathrm{m} / \mathrm{s})$ y VAP $(18.9 \pm 3.2 \mu \mathrm{m} / \mathrm{s})$. Los parámetros cinéticos en semen crioconservado fuero el dilutor 2 VCL $(8.4 \pm 1.4 \mu \mathrm{m} / \mathrm{s})$, el dilutor $1(7.2 \pm 1.2 \mu \mathrm{m} / \mathrm{s})$, VSL el dilutor 2 (3.4 \pm 0.8 $\mu \mathrm{m} / \mathrm{s})$, dilutor $1(2.9 \pm 0.7 \mu \mathrm{m} / \mathrm{s})$, VAP dilutor $2(5.8 \pm 1.0 \mu \mathrm{m} / \mathrm{s})$, dilutor $1(4.8 \pm 0.8 \mu \mathrm{m} / \mathrm{s})$. Se concluye que el dilutor 2 constituye una alternativa más para la crioconservación de semen de alpaca.

\section{Abstract}

Cryopreservation of alpaca sperm is important for genetic improvement. The purpose of this work was to evaluate the sperm kinetic parameters in fresh semen and cryopreserved in dilutors, using the CASA team. 3 male alpacas were used, which were trained for a month in the semen collection with the artificial vagina. The sperm was frozen using two diluents (commercial AndroMed) and (commercial AndroMed, citric acid, gentamicin and glycerol). The semen had the following characteristics, volume $1.5 \pm 0.7 \mathrm{~mL}$, viscosity $5.9 \pm 1.5$, pH 7.1, with an alkalinity tendency and the color that predominated was the translucent white. The averages of kinetic parameters found in fresh semen were VCL $(25.8 \pm 4.2 \mu \mathrm{m} / \mathrm{s})$, VSL $(10.3 \pm 2.5 \mu \mathrm{m} / \mathrm{s})$ and VAP $(18.9 \pm 3.2 \mu \mathrm{m} / \mathrm{s})$. Kinetic parameters in cryopreserved semen showed that dilutor $2 \mathrm{VCL}(8.4 \pm 1.4 \mu \mathrm{m} / \mathrm{s})$, dilutor $1(7.2 \pm 1.2 \mu \mathrm{m} / \mathrm{s})$, VSL dilutor $2(3.4 \pm 0.8 \mu \mathrm{m} / \mathrm{s})$, dilutor $1(2.9 \pm 0.7 \mu \mathrm{m} / \mathrm{s})$, VAP dilutor $2(5.8 \pm 1.0 \mu \mathrm{m} / \mathrm{s})$, dilutor $1(4.8 \pm 0.8 \mu \mathrm{m} / \mathrm{s})$. It is concluded that dilutor 2 is another alternative for cryopreservation of alpaca semen.

\section{Key words: \\ Semen, \\ CASA, \\ evaluation, \\ cryopreservation, \\ alpaca,}




\section{Introducción}

La fisiología reproductiva (FR) presenta una serie de desafíos en la recolección de semen (CS) de camélidos sudamericanos (CSA), incluidos el apareamiento, postura, duración de la cópula, deposición intrauterina y eyaculación durante la cópula ${ }^{1}$. Se han dado un sinfín de métodos para CS de alpacas y llamas incluyendo vagina artificial (VA) ${ }^{2-6}$, aspiración epididimaria ${ }^{7}$, electroeyaculación ${ }^{6,7}$, condones intravaginales ${ }^{7}$, esponjas intravaginales ${ }^{8}$.

La CS con preservativos intravaginales y esponjas interfiere con cópula, resultando una eyaculación incompleta, y el semen frecuentemente está contaminado con sangre, células epiteliales, que no representan la eyaculación general, ya que la mayoría del semen se deposita en el útero ${ }^{8}$.

La aplicación de tecnologías de reproducción asistida (TRA) en la industria ganadera moderna, particularmente la inseminación artificial (IA), fertilización in vitro (FIV) y la transferencia de embriones (TE), contribuyen virtualmente a acelerar la mejora genética en diferentes especies domésticas. Sin embargo, la utilización exitosa de estas técnicas, depende de las características biofísicas de los eyaculados obtenidos, y su capacidad para procesamiento con pérdida mínima de potencial de fertilización espermática $^{9}$.

El procesamiento del semen y la criopreservación, se están convirtiendo en un requisito previo a la aplicación de TRA en camélidos, el desarrollo constante de IA con esperma congelado-descongelado requiere esfuerzos para mejorar la calidad de las técnicas de procesamiento de semen. Uno de los principales desafíos que restringe el desarrollo de TRA en camélidos es la naturaleza altamente viscosa del plasma seminal ${ }^{10,11}$.
Durante la última década, se han utilizado diferentes técnicas para mejorar las propiedades reológicas del semen y mejorar la motilidad de los espermatozoides (spz) en CSA. Algunos investigadores aplicaron licuefacción mecánica, agitación ${ }^{5}$, agitación ${ }^{12,13}$, punción y pipeteo ${ }^{14}$ y centrifugación ${ }^{15}$, últimamente, exposición a ultrasonido con éxito para eliminar viscosidad y mejorar la preservación enfriada a corto plazo del semen de dromedario ${ }^{11}$. Otros autores complementaron el diluyente de semen con diferentes enzimas a fin de eliminar viscosidad, uso de catalizadores de descomposición con peróxido de hidrógeno ${ }^{16}$, catalizadores de descomposición de carbohidratos de cadena larga ${ }^{17,18}$, enzimas proteolíticas $^{14,15,19-22}$.

Avances recientes del análisis de semen asistido por computadora (ASAC) demostraron su capacidad para una estimación precisa de función espermática y heterogeneidad en comparación con la evaluación tradicional del semen ${ }^{23}$. ASAC garantiza la detección de cambios sutiles en movimiento espermático, velocidad y morfología, que confieren una discriminación decisiva entre tratamientos en estudios de procesamiento de semen ${ }^{24}$. Por lo tanto, los centros de IA, especialmente para los humanos, adoptan ASAC para aumentar la objetividad en determinación de la motilidad del esperma ${ }^{25}$.

En consecuencia, la investigación actual tuvo como objetivo determinar los parámetros cinéticos de espermatozoides de alpaca (Vicugna pacos L.) en semen fresco y crioconservado.

\section{Materiales y métodos}

El presente trabajo de investigación, se realizó en el Laboratorio de Biotecnología Reproductiva Animal 
de la Unidad Académica Campesina Tiahuanacu de la Universidad Católica Boliviana "San Pablo", ubicada en la Comunidad de Achaca de la Tercera Sección Municipal de Tiahuanacu, Provincia Ingavi del Departamento de La Paz, a una distancia de 72 $\mathrm{km}$ de la ciudad de La Paz, a una altitud de 3873 msnm, entre los paralelos, $18^{\circ} 20^{\prime}$ y $16^{\circ} 40^{\prime}$ Latitud Sur y $68^{\circ} 35^{\prime}$ y $69^{\circ} 15^{\prime}$ Longitud Oeste ${ }^{26}$.

Selección y adquisición de las alpacas. Se adquirió y seleccionó alpacas de diferentes tamas del Municipio Catacora de la Provincia José Manuel Pando, machos de 2 a 4 años de edad, bajo los siguientes criterios: de condición corporal aceptable, testículos uniformes con consistencia, elasticidad y adherencia prepucial liberada completamente, libre de laceraciones, cicatrices, inflamaciones y otros. Se eligieron 13 alpacas machos, de las cuales se prefirieron 5 machos ya que manifestaron interés de copula.

Desparasitación y vitaminación. Administrados con: desparasitante PARAMEC 20 LA y FOSFOVIT.

Alimentación. Con una dieta a base de forraje: pastos nativos, con suplementos de heno de cebada y alfalfa.

Adiestramiento de las alpacas machos. Se realizaron tres sesiones de colecta en una semana, por un mes, antes del período experimental para su acostumbramiento a la cópula en la VA y a la habituación de la presencia del operador que realizó la colecta del semen, utilizando como estímulo la alpaca hembra, esto con el fin de garantizar que los animales respondan satisfactoriamente durante el periodo de la investigación.

Preparación de los materiales y ambiente. Se desinfecto el ambiente de evaluación con alcohol al 70 $\%$, los materiales se esterilizaron mediante irradiación con luz Ultra Violeta con una intensidad de $20 \mu \mathrm{W} / \mathrm{cm}^{2}$, durante $12 \mathrm{~h}$, para evitar la contamina- ción en la muestra, el ambiente se atempero a $37^{\circ} \mathrm{C}$, con el fin de evitar que los spz sufran un shock térmico al momento de ser evaluados.

Preparado de dilutores comerciales. Se realizó el preparado de dos dilutores comerciales dilutor 1 : AndroMed $3 \mathrm{~mL}$ y agua destilada $6 \mathrm{~mL}$. Dilutor 2: AndroMed $3 \mathrm{~mL}$, ácido cítrico $0.14 \mathrm{~mL}$, gentamicina $0.4 \mathrm{~mL}$, glicerol $1.4 \mathrm{~mL}$, y agua destilada $6 \mathrm{~mL}$, luego se procedió a la refrigeración a $4{ }^{\circ} \mathrm{C}$.

Colección de semen. Para la colecta de semen de alpaca se utilizó el método de colección de VA, con la intervención del operador para la desviación del pene prepucio, la colecta duró aproximadamente 15 a 25 min, pasado este tiempo se retiró al macho y se procedió a coleccionar el eyaculado cuidadosamente evitando contacto con la luz solar y el viento.

Evaluación macroscópica del semen. i) color. Se evaluó directamente del tubo colector por observación directa, considerando las tonalidades básicas del blanco, blanco lechoso, blanco cremoso y blanco cristalino ${ }^{27}$. ii) volumen. Se determinó mediante la medición en tubos de recolección graduados. iii) pH. Se determinó con la ayuda de papel tornasol (indicador de $\mathrm{pH}$ ). iv) filancia. Se tomó $10 \mu \mathrm{L}$ de eyaculado en un porta objeto para ser llevado frente a un filanciometro y con la ayuda de una aguja se estiró el semen y se observó la altura de hilo de la filancia en centímetros ${ }^{28}$.

Evaluación microscópica del semen. i) motilidad espermática. Se colocó $10 \mu \mathrm{L}$ de semen en un porta objeto atemperado a $37{ }^{\circ} \mathrm{C}$, para realizar la lectura en un microscopio a objetivo 40X, contando y valorando los spz que se mueven de forma rectilínea. ii) evaluación de los parámetros cinéticos de los spz de semen fresco. Se utilizó el equipo ASAC, se tomó una muestra de $10 \mu \mathrm{L}$ del eyaculado y se depositó en un porta objeto atemperado para ser llevado al equipo ASAC para la evaluación de los parámetros 
cinéticos: Velocidad curvilínea (VCL), Velocidad rectilínea (VSL) y la Velocidad promedio (VAP).

Incorporación del dilutor. Posterior a la evaluación de la motilidad de los spz, se incorporó el dilutor 1 y 2, a los tubos falcón que contenía el eyaculado.

Congelación de semen. $i$ ) descenso de la temperatura. El semen diluido se sometió a reducción de temperatura a $5{ }^{\circ} \mathrm{C}$, para que el crioprotector vaya ingresando y protegiendo a la célula, para que al momento del congelado no sufran shock térmico. El tiempo que estuvo en refrigeración fue de $2 \mathrm{~h}$, girando cada $0.5 \mathrm{~h}$ a un ángulo de $90^{\circ}$.

Identificación de las pajillas. Se codificó las pajillas, identificándolos por: especie, macho donador del semen, fecha y el dilutor utilizado.

Empajillado de semen. Transcurrido el tiempo establecido se prosiguió al empajillado del semen diluido, para esto se utilizó una jeringa modificada boca ancha para el encaje de las pajillas, seguidamente se acoplo las pajillas codificadas, subsiguientemente se succionó el semen dejando un espacio de aire de 1 $\mathrm{cm}$ en cada pajilla.

Sellado de la pajuela. Cargado la pajilla con semen diluido, se prosiguió con el sellado y posteriormente se mantuvo refrigerado a una temperatura de $5{ }^{\circ} \mathrm{C}$ antes de la congelación.

Crioconservación. Se realizó manualmente, con el método de congelado a vapor de nitrógeno, en un recipiente criogénico modificado, se retiró las pajuelas del refrigerador y se depositó en la rejilla del criogénico, seguidamente se incorporó nitrógeno al recipiente y se sumergió las pajuelas al vapor del nitrógeno a una distancia de $4 \mathrm{~cm}$ del nitrógeno líquido por un lapso de $10 \mathrm{~min}$, pasado este tiempo se sumergió y se mantuvo la rejilla con pajuelas a una distancia $1 \mathrm{~cm}$ distanciado del nitrógeno líquido durante $5 \mathrm{~min}$, transcurrido este tiempo se sumergió poco a poco la rejilla con las pajuelas al nitrógeno líquido hasta sumergirlas por completo. Las pajue- las fueron almacenadas en el tanque de nitrógeno líquido a una temperatura de $-196^{\circ} \mathrm{C}$.

Descongelado de pajuelas. Las pajuelas se descongelaron en baño maría a una temperatura de $38^{\circ} \mathrm{C}$, por un tiempo de 1 min la muestra se llevó en el portaobjeto para evaluar la motilidad de los spz.

Evaluación de los parámetros cinéticos de los espermatozoides crioconservados. Evaluado y observado la motilidad de los spz se evaluó los parámetros cinéticos de los spz usando el equipo ASAC, para la evaluación de los siguientes parámetros cinéticos: VCL, VSL y VAP.

\section{Análisis estadístico}

Factor de estudio: machos 1, 2 y 3, dilutores: 1 (Andromed comercial) 2 (Andromed + ácido cítrico + gentamicina + glicerol)

Variables de respuesta. Características macroscópicas del semen fresco: $\mathrm{pH}$, color, volumen, filancia.

Parámetros cinéticos del semen fresco. VCL, VSL, VAP. Parámetros cinéticos de semen post congelados. VCL, VSL, VAP.

Diseño experimental. Para el análisis macroscópicas y los parámetros cinéticos en semen fresco se utilizó el programa estadístico SAS, se obtuvo: promedio, coeficiente de variación y desviación estándar. Para analizar el efecto de los machos y dilutores en la crioconservación de semen sobre los parámetros cinéticos, se utilizó diseño completamente al azar con arreglo bifactorial el modelo estadístico es el siguiente:

\section{$\mathbf{Y i j n}=\mu+\boldsymbol{\alpha}_{\mathbf{i}}+\boldsymbol{\beta} \mathbf{j}+(\boldsymbol{\alpha \beta}) \mathbf{i j}+\varepsilon_{\mathbf{i j n}}$}

Yijn $=$ Observación cualquiera. $\boldsymbol{\mu}=$ Media general del experimento. $\boldsymbol{\alpha}_{\mathbf{i}}=$ Efecto del i-ésimo machos. $\boldsymbol{\beta} \mathbf{j} \quad=$ Efecto del $\mathbf{j}$-ésimo dilutores. $(\boldsymbol{\alpha} \boldsymbol{\beta}) \mathbf{i j}=$ Interacción del i-ésimo machos con la j-ésimo dilutores. Interacción machos $\mathrm{x}$ dilutores. $\mathbf{E}_{\mathbf{i j n}}=$ Error experimental

Pruebas de promedios. Las diferencias estadísticas encontradas fueron analizadas mediante la prueba de comparación de medias DUNCAN a un nivel de significancia del $5 \%(\alpha=0.05)$. 


\section{Resultados}

Tabla 1 Valores de las características macroscópicas del semen fresco de alpaca colectados por vagina artificial

\begin{tabular}{cccccc}
\hline Características Macroscópicas & $\mathbf{N}$ & Promedio & DS \pm & Max & Min \\
\hline Color & 20 & Blanco translucido & - & - & - \\
pH & 20 & 7.1 & 0.2 & 7.4 & 6.7 \\
Volumen $(\mathrm{mL})$ & 20 & 1.5 & 0.7 & 2.5 & 1.5 \\
Filancia $(\mathrm{cm})$ & 20 & 5.9 & 1.5 & 6 & 1.8 \\
\hline
\end{tabular}

Tabla 2 Valores de parámetros cinéticos de espermatozoides del semen fresco evaluados mediante el equipo ASAC

\begin{tabular}{cccccc}
\hline Parámetros & Muestra & $\mathbf{N}$ & Promedio & SD \pm & C.V. \\
\hline \multirow{3}{*}{ Velocidad curvilínea $(\boldsymbol{\mu m} / \mathbf{s})$} & macho 1 & 20 & 24.0 & 3.8 & 15.6 \\
& macho 2 & 20 & 24.1 & 3.6 & 15.1 \\
& macho 3 & 20 & 29.3 & 5.2 & 17.7 \\
\cline { 2 - 5 } Velocidad rectilínea $(\boldsymbol{\mu \mathrm { m }} / \mathbf{s})$ & macho 1 & 20 & 8.0 & 1.8 & 22.6 \\
& macho 2 & 20 & 7.8 & 1.8 & 22.9 \\
\cline { 2 - 6 } & macho 3 & 20 & 15.9 & 4.0 & 25.0 \\
\hline \multirow{2}{*}{ Velocidad promedio $(\boldsymbol{\mu m} / \mathbf{s})$} & macho 1 & 20 & 11.5 & 2.2 & 19.4 \\
& macho 2 & 20 & 11.3 & 2.4 & 21.1 \\
& macho 3 & 20 & 23,9 & 3.5 & 10,5 \\
\hline
\end{tabular}

Tabla 3 Valores de parámetros cinéticos de semen crioconservado evaluados mediante el equipo ASAC

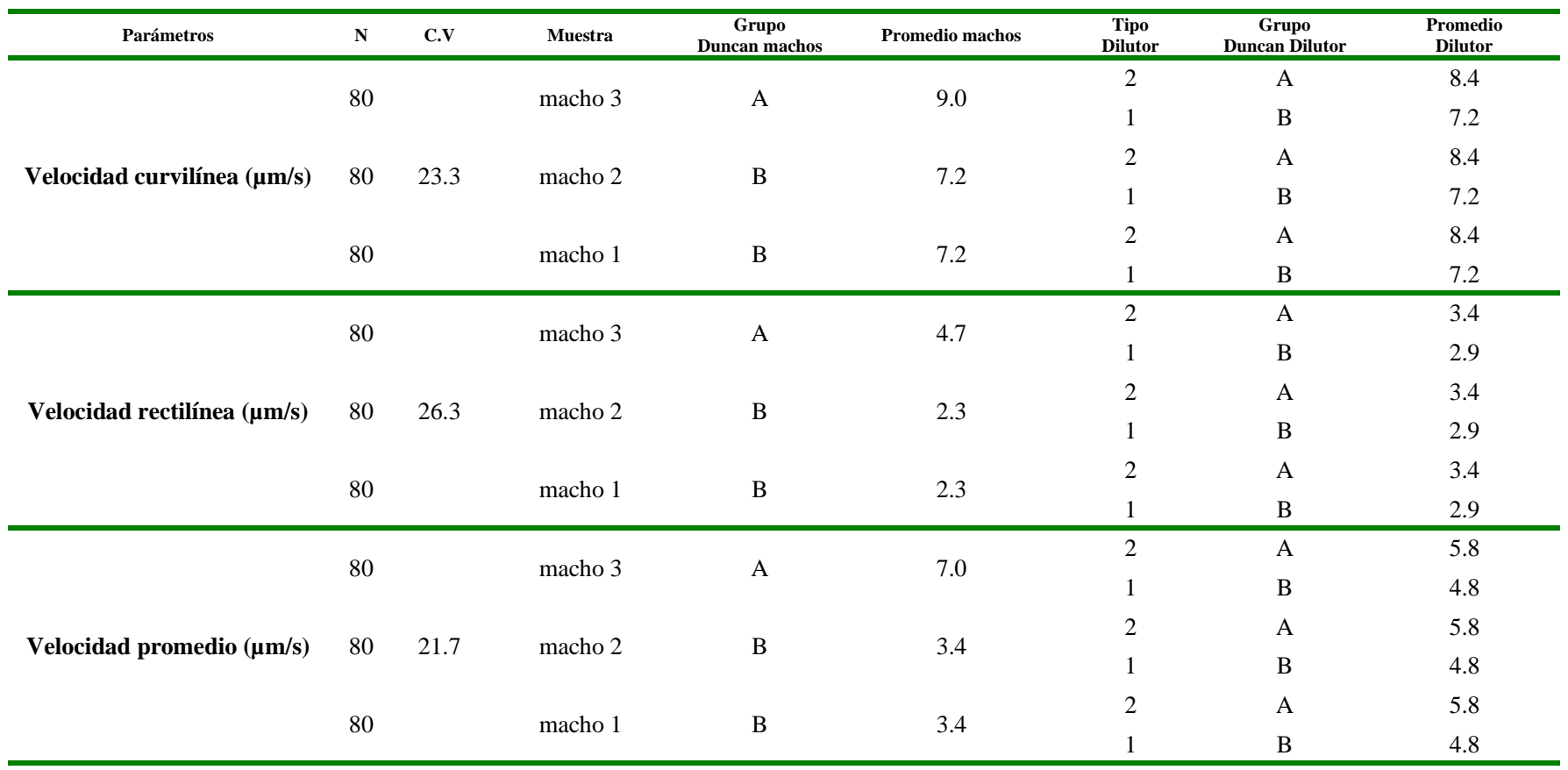


Figura 1 Comparación de las velocidades cinéticas espermáticas del semen fresco colectado por vagina artificial

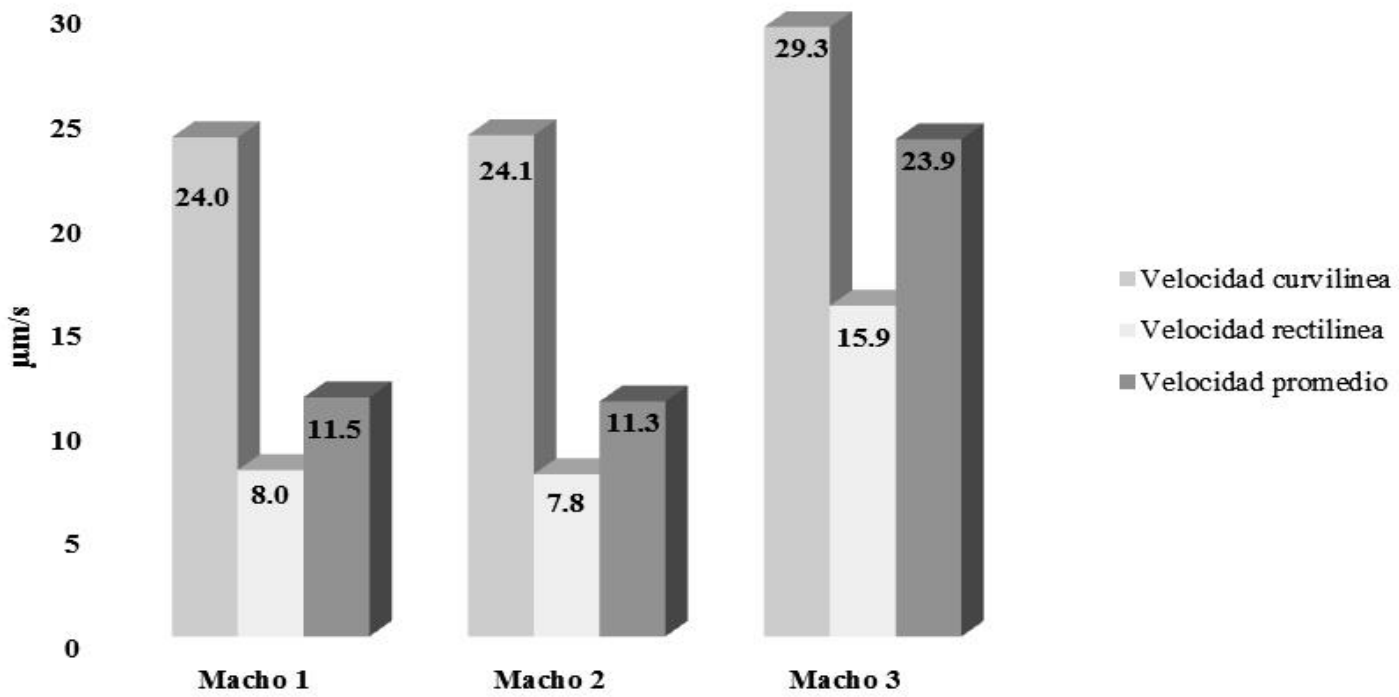

Figura 2 Comportamiento de las velocidades espermáticas por efecto de crioconservación en dos dilutores

macho 1 macho 2 macho 3

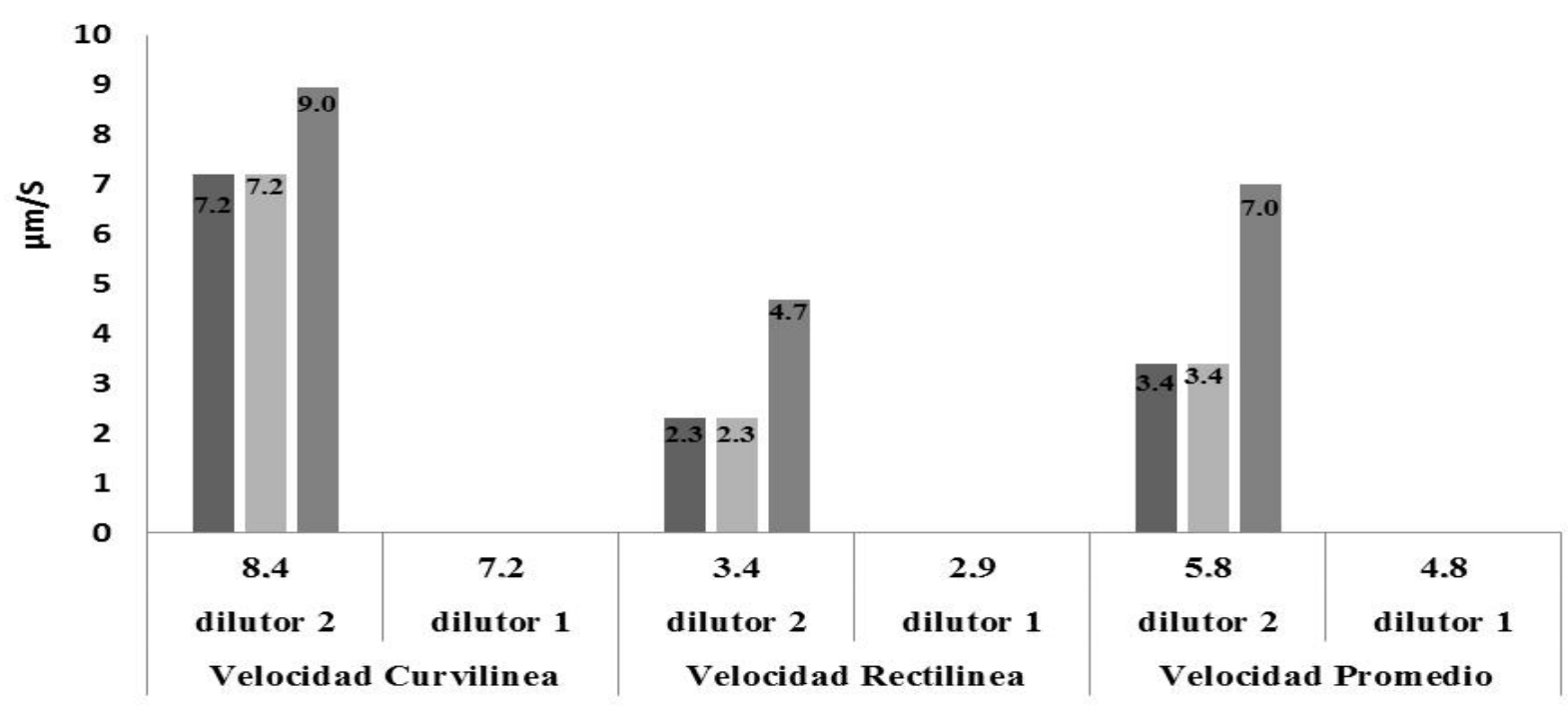

\section{Discusión}

Los procedimientos para el procesamiento del semen difieren en especies y más aún en los CSA, ya que presentan problemas en el manejo, congelación, aplicación de dilutores, y eliminación de contaminantes que presentan como células epiteliales, sangre y otros, en su evaluación macroscópica, el volumen fue superior, ya que los machos fueron jóvenes, 2 a 4 años de edad, presentaban condición cor- 
poral buena, también puede atribuirse a la temperatura de la VA, que fue similar al de la hembra, en tanto hay reportes, con volumen promedio por método de electroeyaculación de $1.58 \mathrm{~mL}^{29}$, se menciona que volumen colectado en VA modificada incorporada al maniquí de alpaca $1.03 \mathrm{~mL}$, con hembras receptivas $1.73 \mathrm{~mL}^{30}$. El volumen promedio de semen de las colectas realizadas fue $1.5 \mathrm{~mL}$, que está entre los reportados por los anteriores autores. Investigaciones realizadas, mencionan que el volumen obtenido por el método de maniquí de alpaca en la época lluviosa $1.28 \mathrm{~mL}$ y en época seca $1.12 \mathrm{~mL}^{31}$.

La filancia del semen fresco de alpaca presento un promedio de $5.9 \mathrm{~cm}$, mientras que Ordóñez et al. ${ }^{29}$, obtuvo un promedio de $1.6 \mathrm{~cm}$, con un macho con experiencia en empadre para la reproducción, una filancia de $1 \mathrm{~cm}$, al mismo tiempo indica que mientras más baja sea la filancia existe mayor concentración de $\mathrm{spz}^{31}$.

La viscosidad del semen se debe a las mucoproteinas secretadas por las glándulas bulbouretrales que constituyen el $80 \%$ del eyaculado, la alta viscosidad del semen de llama, se atribuye al fosfato de espermina y proteínas, estas son secretadas por la glándula prostática ${ }^{32}$, el extracto de piña al presentar la enzima bromelina, una endopeptidasa, rompe las uniones peptídicas las que producen una ruptura a nivel de la lisina, alanina y tiroxina ${ }^{33}$.

Un pH en época de lluvia 7.59, en época seca 7.70, por lo que menciona que existe diferencias entre las épocas de colección ${ }^{31}$. El $\mathrm{pH}$, normalmente varía entre 7.2-8.2, debe medirse antes de una hora de la eyaculación, ya que se libera $\mathrm{CO}_{2}$ que modifica a valores fuera de este rango pueden significar una infección genital, si los valores están por debajo de 7, la causa puede deberse a una obstrucción de los conductos eyaculadores o bien a una agenesia de 23 los vasos deferentes. Por otro lado, si los valores del $\mathrm{pH}$ del semen se encuentran por encima de 8.2, la causa se debe a que el plasma seminal haya liberado dióxido de carbono $\mathrm{CO}_{2}$ de forma continua provocando de esta forma un aumento del $\mathrm{pH}^{31}$.

El promedio de $\mathrm{pH}$ del semen en la presente investigación fue 7.1, siendo inferior al anterior autor, se asume que el pH sufrió cambios y modificación en el momento de la colecta por la presencia de $\mathrm{CO}_{2}$. Dado que los spz en condiciones aerobias al momento de la recogida de tubos colectores, descomponen la fructosa en ácido láctico, el pH disminuye es posible, el tiempo transcurrido desde la recogida a su determinación. Por otro lado, el semen colectado por medio de VA usualmente tiene menor $\mathrm{pH}$, que el colectado por medio del masaje rectal. En este último método, el pH oscila en un rango de 7.5 a 8. Eyaculaciones sucesivas tienden a aumentar el $\mathrm{pH}$, y el semen que no contiene spz, provenientes de toros que tienen inflamación de los epidídimos, o el semen obtenido por medio de la vasectomía, tienden a tener niveles de $\mathrm{pH}$ mayores ${ }^{34}$.

Los valores de VCL de $24.0 \mu \mathrm{m} / \mathrm{s}$ del macho 1 , el macho 2, $24.1 \mu \mathrm{m} / \mathrm{s}$ alcanzando valores de 29.3 $\mu \mathrm{m} / \mathrm{s}$ el macho 3 , la diferencia pudo influenciar en el obtenido de los resultados, podría ser por el factor de temperatura. Los valores de coeficientes de variación para cada macho en la evaluación de la VCL varían desde un $15.6 \%$ para el macho 1 y un $15.1 \%$ para el macho 2 , sucesivamente a $17.7 \%$ para el macho 3, se podría decir que los datos menores son más confiables para realizar la crioconservación.

En la figura 1, señala parámetros cinéticos estadísticos, reportan las características de los eyaculados obtenidos en 3 alpacas machos en semen fresco sin dilutor, pero incorporado bromelina para disminuir la filancia, la mayor parte de las características 
seminales analizadas presentaron coeficientes de variación moderados, los que están dentro de los rangos de $25.0 \%$ a $14.8 \%$.

Los resultados en promedios en la VCL tuvieron como promedio de $(24.0 \pm 3.8,24.1 \pm 3.6$ y $29.3 \pm 5.2$ $\mu \mathrm{m} / \mathrm{s})$, en la VSL se obtuvo promedios de $(8.0 \pm 1.8$, $7.8 \pm 1.8$ y $15.9 \pm 4.0 \mu \mathrm{m} / \mathrm{s}$ ) y en la VAP un $(11.5 \pm 2.2,11.3 \pm 2.4$ y $23.9 \pm 3.5 \mu \mathrm{m} / \mathrm{s}$. Sin embargo, hay reportes promedios $68.2 \pm 17.7 \mu \mathrm{m} / \mathrm{s}$ en la VCL, $19.9 \pm 8.1 \mu \mathrm{m} / \mathrm{s}$ en la VSL y $38.2 \pm 13.4 \mu \mathrm{m} / \mathrm{s}$ en la VAP, en semen fresco, la investigación hecha por este autor fue realizada en alpaca macho de 7 años de edad, agregó el dilutor Triladyl® al semen ${ }^{29}$. En semen fresco de conejo, utilizando el sistema ASAC, obtuvo una VCL de 84.9 $\pm 1.1,41.7 \pm 0.9$ $\mu \mathrm{m} / \mathrm{s}$ de VR $56.1 \pm 0.9 \mu \mathrm{m} / \mathrm{s}$ de VAP incorporando el dilutor, $0.25 \mathrm{M}$ de Tris[hidroximetil]aminometano, $88 \mathrm{mM}$ de ácido cítrico, y $47 \mathrm{mM}$ de D-glucosa, al que se adicionaron los crioprotectores dimetilsulfóxido y sacarosa a una concentración de $3.5 \mathrm{M}$ y $0.1 \mathrm{M}$ respectivamente ${ }^{35}$.

La VCL en nuestra investigación presento $24.0 \pm 3.8,24.1 \pm 3.6$ y $29.3 \pm 5.2 \mu \mathrm{m} / \mathrm{s}$, estos datos resultan ser menores a los descritos por Ordóñez et al. ${ }^{29}$ que reporta un $68.23 \pm 17.7 \mu \mathrm{m} / \mathrm{s}$, Ferrian ${ }^{35}$ de $84.9 \pm 1.1 \mu \mathrm{m} / \mathrm{s}$, las diferencias que existe entre los resultados se podría asumirse que estos autores utilizaron dilutores en el semen colectado, en cambio en el presente trabajo no se agregó al semen ningún dilutor para la capacitación de los spz.

Nuestros datos de VSL $8.0 \pm 1.8,7.8 \pm 1.8 \mathrm{~s}$ y $15.9 \pm 4.0 \mu \mathrm{m} / \mathrm{s}$, estos efectos mínimos de los resultados en comparación a los datos reportados por Ordóñez et al. ${ }^{29}$ de $38.2 \pm 13.4 \mu \mathrm{m} / \mathrm{s}$ y a los de Fe$\operatorname{rrian}^{35}$ indica que reporto $41.7 \pm 0.9 \mu \mathrm{m} / \mathrm{s}$, las diferencias que existentes entre datos se debe a que los spz probablemente sufrieron una híperactivación para continuar con la motilidad progresiva.
La VAP en semen fresco de alpaca evaluado con el equipo ASAC reporto un $11.5 \pm 2.2 \mu \mathrm{m} / \mathrm{s}, 11.3 \pm 2.4$ $\mu \mathrm{m} / \mathrm{s}$ y $23.9 \pm 3.5 \mu \mathrm{m} / \mathrm{s}$, son menores a los citados por Ordóñez et al. ${ }^{29}$ con valores promedio $38.2 \pm 13.4 \mu \mathrm{m} / \mathrm{s}$, con respecto a lo observado por Cucho et al. ${ }^{36}$ en semen de zorro con promedio $52.5 \pm 42.2 \mu \mathrm{m} / \mathrm{s}$, la discrepancia que existe entre resultados se debe a que la velocidad de los spz que no tuvieron un desplazamiento extenso en su trayectoria, en cambio también se observa que existe una baja actividad espermática, como lo manifiestan los datos de las variables de velocidad (VCL, VSL, VAP). Al observar los valores medios de la cinética espermática de cada uno de los machos alpaca, podemos advertir que también experimentan la misma tendencia, por tanto, los índices de linealidad, rectitud y oscilación serán superiores en valores.

Los análisis de varianza para las velocidades de los spz a efecto de ser crioconservados con los dilutores 1 (AndroMed comercial) y el dilutor 2 (AndroMed comercial, gentamicina, ácido cítrico y glicerol), expresan diferencias entre los parámetros cinéticos (VCL, VSL y VAP), en los 3 machos, las diferencias significativas $(\mathrm{p}<0.05)$, tabla 3 .

En la figura 2, se observa el comportamiento de las velocidades (curvilínea, rectilínea y promedio) en semen de alpacas crioconservado con los dilutores 1 y 2. Como en la tabla 3 , el dilutor 2 mantiene resultados superiores con respecto al dilutor 1 que presento descenso en la motilidad de los spz.

Los resultados con el dilutor 2, tuvieron como promedio VCL el macho $3(9.0 \mu \mathrm{m} / \mathrm{s})$, el macho $2(7.2$ $\mu \mathrm{m} / \mathrm{s})$ y el macho $1(7.2 \mu \mathrm{m} / \mathrm{s})$, el dilutor 2 para los 3 machos fue de 8.4 y el dilutor 1 logró un promedio de 7.2 para los 3 machos. Así mismo VCL obtuvo un coeficiente de variación del $23.3 \%$ este valor fue para los 3 machos, 
La VSL promedios fueron, macho $3(4.7 \mu \mathrm{m} / \mathrm{s})$, macho $2(2.3 \mu \mathrm{m} / \mathrm{s})$ y macho $1(2.3 \mu \mathrm{m} / \mathrm{s})$, con un coeficiente de variación para los 3 machos de 26.3 $\%$, los promedios entre dilutores se puede observar la diferencia que existe ya el dilutor 2 obtuvo en promedio un 3.4 en los 3 machos esto resulto ser superior en los comparación al dilutor 1 que tuvo un promedio de 2.9 para los 3 machos y la VAP en el macho $3(7.0 \mu \mathrm{m} / \mathrm{s})$, macho $2(3.4 \mu \mathrm{m} / \mathrm{s})$ y macho $1(3.4 \mu \mathrm{m} / \mathrm{s})$, los promedios con el dilutor 2 fue 5.8 y el dilutor 14.8 , con un coeficiente de variación para los 3 machos de $21.7 \%$ en la VAP también se puede distinguir claramente la diferencia en los valores de los dilutores.

Medina et al. ${ }^{37}$, Farrel et al. ${ }^{38}$, menciona que los resultados en semen de bovino crioconservado en la VCL fue $30.4 \mu \mathrm{m} / \mathrm{s}$, la disminución de la motilidad de los spz se deba a que sufrieron una híperactivación al momento de la congelación pudo haber un daño en el acrosoma.

Ancco et al..$^{39}$, obtuvo un promedio de VCL $73.2 \pm 9.1 \mu \mathrm{m} / \mathrm{s}$, VSL $26.1 \pm 3.5 \mu \mathrm{m} / \mathrm{s}$, VAP $41.4 \pm 4.6$ $\mu \mathrm{m} / \mathrm{s}$, en semen empajillado post-descongelado de bovinos pero antes de la centrifugación con Percoll. Tejerina ${ }^{40}$, menciona que en la VCL sin cafeína. $87.8 \pm 14.5 \mu \mathrm{m} / \mathrm{s}$, con cafeína $104.58 \pm 12.61 \mu \mathrm{m} / \mathrm{s}$, VSL sin cafeína $65.1 \pm 12.4 \mu \mathrm{m} / \mathrm{s}$, con cafeína $85.7 \pm 10.6 \mu \mathrm{m} / \mathrm{s}$, VAP sin cafeína $71.6 \pm 12.9 \mu \mathrm{m} / \mathrm{s}$, con cafeína $91.9 \pm 11.1 \mu \mathrm{m} / \mathrm{s}$, estos datos fueron evaluados en semen de verraco para lo cual se trabajó con una docena de verracos.

La pérdida del porcentaje de motilidad desde la colección de semen en la refrigeración, depende de la temperatura final, la velocidad de disminución de la temperatura, la relación de la dilución e intervalo de tiempo que los spz se hallan expuestos a la temperatura final de refrigeración ${ }^{41}$.
En nuestra investigación la comparación realizada de VCL valores inferiores de motilidad incorporados con 2 diferentes dilutores, (9.0, 7.2 y $7.2 \mu \mathrm{m} / \mathrm{s}$ ), ocurrieron cambios en el descenso VCL de los spz en comparación del dilutor 2 con el dilutor 1 como se observa en la tabla 3 y en comparación con los resultados reportados por el semen de bovino crioconservado una VCL fue $30.4 \mu \mathrm{m} / \mathrm{s}^{37,38}$.

Los spz incorporados con dilutores para la congelación, reaccionaron de forma completamente distinta a los procesos de la crioconservación, debido a que sufren daños en el metabolismo espermático y afecto a dos estructuras muy importantes para el desarrollo de la motilidad espermática: la mitocondria y el flagelo.

En la VCL en semen crioconservado de alpaca, los promedios, 4.7, 2.3 y $2.3 \mu \mathrm{m} / \mathrm{s}$, los datos similares entre el macho 1 y 2 después de la descongelación puede deberse a que los 2 machos son de misma edad y el macho 3 tiene una edad mayor.

Según Benon \& Linet ${ }^{42}$, reportan que hay un aumento significativo en amplitud del desplazamiento lateral de la cabeza (ALH), VCL y en menor proporción VSL. Esto parece caracterizar por un aumento significativo en el esperma hiperactivo causada por los dilutores. En comparación con el anterior autor mencionado se puede observar la disminución de la VSL en los spz de alpacas probablemente la reacción de los spz se podría dar a consecuencia de la incorporación del dilutor al semen de alpaca y debido a que no ingresó el dilutor por completo al interior de los spz en el momento de la refrigeración y se haya formado cristales de hielo. Los datos obtenidos en nuestra investigación VAP en semen crioconservado de alpaca fueron, 7.0, 3.4 y $3.4 \mu \mathrm{m} / \mathrm{s}$, debido a la influencia de los diluyentes la disminución de la motilidad espermática es notable, la función que cumplen los diluyentes o crio- 
protectores es sustituir y extraer el agua del citoplasma y así evitar que durante la crioconservación se formen cristales de hielo en el interior de la célula, si no se realiza la deshidratación adecuada de los spz puede producir daños irreversibles al momento de la crioconservación.

Existen alteraciones en spz debido a que al momento de la crioconservación, con valores superiores a los autores mencionados anteriormente, son similares en los spz de los 3 machos alpacas que se sometieron a 2 h de refrigeración, en ese momento sufre un proceso a la que se somete los spz la producción de ATP disminuye y se produce un shock térmico y de esta manera reduce la vitalidad en el tiempo de refrigeración ya que puede existir el estrés oxidativo la que sufren las membranas celulares y de manera que en el transcurso existe la liberación de radicales libres que conllevan a la muerte celular y los spz en la etapa de refrigeración a $5{ }^{\circ} \mathrm{C}$ no cesan su actividad metabólica, al estar inmóviles estas se encuentran en constante producción de ATP mediante el ciclo de Krebs en las mitocondrias.

Las células espermáticas sufren daños severos durante la congelación. Para lograr éxito en dicho proceso el spz deberá sufrir previamente una serie de cambios, englobados bajo la denominación de capacitación, que entre otros fenómenos desencadena un cambio en el patrón cinético que recibe el nombre de "hiperactividad" 43 , caracterizándose por un escaso movimiento progresivo, muy vigoroso y con amplios batidos flagelares ${ }^{44}$.

\section{Conflictos de intereses}

En el manejo de los animales se ha cumplido normas éticas. Esta investigación ha sido autofinanciada por los autores y no genera conflictos de interés.

\section{Agradecimientos}

Al personal del Laboratorio de Biotecnología Reproductiva Animal de la Carrera de Ingeniera Zootécnica (LBRA-CZ). (Universidad Católica Boliviana San Pablo, Bolivia) por su apoyo en el análisis de las muestras evaluadas en la presente investigación. A los Comunarios del Municipio Catacora de la Provincia José Manuel Pando, por proporcionarnos los camélidos para esta investigación.

\section{Aspectos éticos}

Al personal del Laboratorio de Biotecnología Reproductiva Animal de la Carrera de Ingeniera Zootécnica (LBRA-CZ). (Universidad Católica Boliviana San Pablo, Bolivia) por su apoyo en el análisis de las muestras evaluadas en la presente investigación, y respetando las normas de bienestar animal, garantizándose la seguridad sanitaria de los alimentos y la inocuidad alimentaria.

\section{Literatura citada}

1. Bravo PW, Skidmore JA, Zhao XX. Reproductive aspects and storage of semen in Camelidae. Anim Reprod Sci 2000;62(1-3):173-93. DOI: http://doi.org/10.1016/s0378-4320(00)00158-5

2. Bravo PW, Flores D, Ordoñez C. Effect of repeated collection on semen characteristics of alpacas. Biol Reprod 1997a;57(3):520-4. DOI: http://doi.org/10.1095/biolreprod57.3.520

3. Bravo PW, Flores U, Garnica J, Ordoñez C. Collection of semen and artificial insemination of alpacas. Theriogenology 1997b;47(3):619-26. DOI: http://doi.org/10.1016/s0093-691x(97)000 20-4

4. Bravo PW, Moscoso R, Alarcon V, Ordoñez C. Ejaculatory process and related semen character- 
istics. Arch Androl 2002;48(1):65-72. DOI: http: //doi.org/10.1080/014850102753385224

5. Vaughan JL, Galloway D, Hopkins D. Artificial insemination in alpacas (Lama pacos). Barton: A report for the Rural Industries Research and Development Corporation; 2003. RIRDC Publication No 03/104.

6. Giuliano S, Director A, Gambarotta M, Trasorras V, Miragaya M. Collection method, season and individual variation on seminal characteristics in the llama (Lama glama). Anim Reprod Sci 2008; 104(2-4):359-69. DOI: http://doi.org/10.1016/j. anireprosci.2007.02.016

7. McEvoy TG, Kyle CE, Slater D, Adam CL, Bourke DA. Collection evaluation and cryopreservation of llama semen. J Reprod Fertil 1992; 9:48.

8. San Martin M, Copaira M, Zuniga J, Rodreguez R, Bustinza G, Acosta L. Aspects of reproduction in the alpaca. J Reprod Fertil 1968;16(3):395-9. DOI: http://doi.org/10.1530/ jrf.0.0160395

9. Mostafa TH, Abd El-Salaam AM, Elbadry DE, Anour AM. Freezability and DNA integrity of dromedary camel spermatozoa in semen collected by artificial vagina and electro-ejaculator. Egyptian J Anim Prod 2014;51(2):145-55.

10.Skidmore JA, Morton KM, Billah M. Artificial insemination in dromedary camels. Anim Reprod Sci 2013;136(3):178-86. DOI: https://doi.org/10. 1016/j.anireprosci.2012.10.008

11.Rateb SA. Ultrasound-assisted liquefaction of dromedary camel semen. Small Rumin Res 2016; 141:48-55. DOI: http://doi.org/10.1016/j. smarum res.2016.07.005

12.Mosaferi S, Niasari Nisalaji A, Abarghani A, Gharahdaghi AA, Gerami A. Biophysical and biochemical characteristics of bactrian camel semen collected by artificial vagina. Theriogenolo- gy 2005;63(1): 92-101. DOI: http://doi.org/10. 1016/j.theriogenology.2004.03.021

13.Niasari Naslaji A, Mosaferi S, Bahmani N, Gerami A, Gharahdaghi AA, Abarghani A et al. Semen cryopreservation in Bactrian camel (Camelus bactrianus) using SHOTOR diluent: effects of cooling rates and glycerol concentrations. Theriogenology 2007;68(4):618-25. DOI: http://doi.org/10.1016/j.theriogenology.2007.04.0 $\underline{59}$

14.Morton KM, Vaughan J, Maxwell WM. Continued development of artificial insemination technology in alpacas. Rural Industries Research and Development Corporation, Kingston, ACT Australia; 2008.

15.El-Bahrawy KA, El-Hassanien EE, Fateh El-Bab AZ, Zeitoun MM. Semen characteristics of the male camel and its freezability after dilution in different extenders. In: Int Sci Camel Conf, editors. El-Qaseem, KSA; 2006. p. 2037-53.

16. Medan MS, Absy G, Zeidan AE, Khalil MH, Khalifa HH, Abdel Salaam AM et al. Survival and fertility rate of cooled dromedary camel spermatozoa supplemented with catalase enzyme. J Reprod Dev 2008;54(1):84-89. DOI: http://doi. org/10.1262/jrd.19124

17.El-Bahrawy KA. Cryopreservation of dromedary camel semen supplemented with $\alpha$-amylase enzyme. J Camel Pract Res 2010;17(2):211-6.

18. Monaco D, Fatnassi M, Padalino B, Hammadi M, Khorchani T, Lacalandra GM. Effect of $\alpha$ Amylase, Papain, and Spermfluid ${ }^{\circledR}$ treatments on viscosity and semen parameters of dromedary camel ejaculates. Res Vet Sci 2016;105:5-9. DOI: http://doi.org/10.1016/j.rvsc.2016.01.003

19. Shekher C, Vyas S, Purohit GN, Patil NV. Use of collagenase type-1 to improve the seminal char- 
acteristics of dromedary camel semen. Eur J Vet Med 2012;1(1):17-27.

20.Kershaw Young CM, Stuart C, Evans G, Maxwell WM. The effect of glycosaminoglycan enzymes and proteases on the viscosity of alpaca seminal plasma and sperm function. Anim Reprod Sci 2013;138(3-4):261-7. DOI: http://doi. org/10.1016/j.anireprosci.2013.02.005

21. Kershaw Young CM, Evans G, Rodney R, Maxwell W. Papain and its inhibitor E-64 reduce camelid semen viscosity without impairing sperm function and improve post-thaw motility rates. Reprod Fert Dev 2017;29(6):1107-14. DOI: http://doi.org/10.1071/RD15261

22.Mal G, Vyas S, Srinivasan A, Patil NV, Pathak KML. Studies on liquefaction time and proteins involved in the improvement of seminal characteristics in dromedary camels (Camelus dromedarius). Scientifica (Cairo) 2016; 4659358. DOI: http://doi.org/10.1155/2016/4659358

23. Holt WV, O'Brien J, Abaigar T. Applications and interpretation of computer-assisted sperm analyses and sperm sorting methods in assisted breeding and comparative research. Reprod Fert Dev 2007;19(6):709-18. DOI: http://doi.org/10. $\underline{1071 / \mathrm{rd} 07037}$

24.Amann RP, Katz DF. Reflections of CASA after 25 years. J Androl 2004;25(3):317-25. DOI: http://doi. org/10.1002/j.1939-4640.2004.tb02793 $\underline{. \mathrm{X}}$

25.Kathiravan P, Kalatharan J, Karthikeya G, Rengarajan K, Kadirvel G. Objective sperm motion analysis to assess dairy bull fertility using computer-aided system-a review. Reprod Domest Anim 2001;46(1):165-72. DOI: http://doi.org/ 10.1111/j.1439-0531.2010.01603.x

26.Plan de Desarrollo Municipal. Gobierno Autónomo Municipal de Tiahuanacu Estado Plurina- cional de Bolivia departamento de La Paz provincia Ingavi tercera sección; 2011.

27.Pacheco JI, Mamani RH, Deza HW. Colección de semen mediante electroeyaculación de vicuñas mantenidas en cautiverio. Spermova 2011; 1(1): 131-3.

28. Quispe Y. Efecto de tres niveles de glicerol y tres tiempos de refrigeración sobre las características microscópicas de semen post-congelado de llama (Lama glama) [tesis licenciatura]. [Tiahuanacu]: Universidad Católica Boliviana San Pablo; 2003

29. Ordóñez C, Ampuero E, Cucho H, Franco E. Avances en la determinación de la velocidad del espermatozoide de alpaca en semen diluido usando el Integrated Sperm Anaysis System (ISAS). Spermova 2012;2(1):63-4.

30.Dávalos R, Olazábal J. Evaluación de dos formas de colección de semen en Alpacas. Rev Investig Vet Perú 2002;13(2):98-9. DOI: http://doi.org/ 10.15381/rivep.v13i2.7340

31.Huanca T, Mamani RH, Naveros ML, Pacheco JI, Condori N. Variación individual y estacional de las características seminales en la alpaca ( $V i$ cugna pacos L.) Spermova 2011;1(1):98-100.

32.Gonzales V. Efecto de la bulbourectomia y periodicidad de colección en las características macro-microscópicas del eyaculado en llamas de tres edades [tesis licenciatura]. [Tiahuanacu]: Universidad Católica Boliviana San Pablo; 2003.

33. Alan W, Louis K, Andrew N, Alan P, Graig P. Urologia [Internet]. New York: Editorial Medica Panamericana; 2015[citado 22-de octubre de 2018]. Recuperado a partir de: https://www. medicapanamericana.com/materialesComple mentarios/Campbell-10/Campbell.aspx

34. Salisbury GW. Fisiología de la reproducción e inseminación artificial de los bóvidos [Internet]. España: Editorial: Editorial Acribia SA; 1982 [ci- 
tado 22-de octubre de 2018]. Recuperado a partir de: https://www.iberlibro.com/products/isbn/978 $\underline{8420005119 \text { ?cm_sp=bdp-_-ISBN10-_-PLP }}$

35.Ferrian S. Influencia de las características seminales del eyaculado de conejo sobre la calidad espermática post-descongelación [tesis licenciatura]. [Valencia]: Universidad Politécnica de Valencia; 2007 [citado 26 de octubre de 2018]. Recuperado a partir de: https://docplayer.es/ 1099 9811-Influencia-de-las-caracteristicas-semi nalesdel-eyaculado-de-conejo-sobre-la-calidadespermatica-postdescongelacion.html

36.Cucho H, Ordoñez C, Ampuero E, Alarcón V, Quispe H. Estimación de parámetros de movilidad en espermatozoides epididimarios de un zorro andino (Lycalopex culpaeus andinus) mediante el isas®. Spermova 2014;4(1):89-91.

37.Medina Robles VM, Sánchez Carvajal E, Velasco Santamaría YM, Cruz Casallas PE. Crioconservación de semen bovino usando un congelador programable (CL-8800) y determinación de su calidad postdescongelación por medio un sistema de análisis espermático asistido por computador (CASA). Orinoquia 2007;11(1):75-86.

38.Farrell PB, Presicce GA, Brockett CC, Foote RH. Quantification of bull sperm characteristics measured by computer-assisted sperm analysis (CASA) and the relationship to fertility. Theriogenology 1998;49(4):871-9. DOI: https://doi.org /10.1016/S0093-691X(98)00036-3
39.Ancco E, Quispe C, Mellisho E. (2014). Parámetros de cinética espermática antes y después de la selección con gradiente de percoll 45/90 en semen congelado de bovinos. Spermova 2014;4(1): 65-7.

40.Tejerina Ampudia F. Valoración mediante imágenes digitales del semen descongelado de verraco [tesis licenciatura]. [León]: Universidad de León; 2007[citado 26 de octubre de 2018]. Recuperado a partir de: https://dialnet. Unirioja.es/ servlet/tesis?codigo $=26987$

41.Stornelli MC, Tittarelli CM, Savignone CA, Stornelli MA. Efecto de los procesos de criopreservación sobre la fertilidad seminal. Analecta Vet 2005;25(2):28-35.

42.Benon M, Linet $T$. Cinétique spermatique d'hyperactivation : influence du milieu de capacitationSperm hyperactivated motility: influence of the capacitation medium. Gynecol Obstet Biol Reprod (Paris) 2005;34(5):488-92. DOI: https: //doi.org/10.1016/S0368-2315(05)82857-1

43. Ho HC, Suarez SS. Hyperactivation of mammalian spermatozoa: function and regulation. Reproduction 2001;122(4):519-26. DOI: http://doi.org/ 10.1530/rep.0.1220519

44. Yanagimachi R. Fertility of mammalian spermatozoa: its development and relativity. Zigote; 1994;2(4):371-2. DOI: http://doi.org/10.1017/s $\underline{0967199400002240}$

Nota del Editor:

Journal of the Selva Andina Animal Science (JSAAS) se mantiene neutral con respecto a los reclamos jurisdiccionales publicados mapas y afiliaciones institucionales. 